\title{
Treatment of benign intracranial hypertension by dehydrating agents with particular reference to the measurement of the blind spot area as a means of recording improvement
}

\author{
ANTONY JEFFERSON AND JANET CLARK \\ From the Department of Neurological Surgery, The Royal Infirmary, Sheffield
}

SYNOPSIS A series of 30 patients (28 females : 2 males) presenting with benign intracranial hypertension is reported. It is shown that the papilloedema may be resolved in as little as six weeks by the use of dehydration therapy. Evidence is presented that this method can restore to normal a visual acuity which is as low as $6 / 24$ or even $6 / 36$. It is concluded that surgery is never required in the management of this condition. It is strongly recommended that serial blind-spot measurements should be used routinely to assess the effectiveness of therapy. Fluorescein angiography has a place in diagnosis among a small proportion of these patients.

The existence of papilloedema in the absence of an intracranial mass poses problems which are no less intriguing for the diagnostician than to the person responsible for their treatment. One of the interesting features of so-called 'benign intracranial hypertension' is that, while it is harmless to life, it can be devastating to vision for, at its worst, bilateral permanent blindness may ensue.

The purpose of this paper is to show the value of measuring the blind spots; also to record the results of the various methods used in the management of this condition in a series of 30 patients treated in this department over the past 17 years.

We propose a simple formula which may be easily employed to convert the measurements of a blind spot (as charted on a Bjerrum screen) into an area. The convenience of working with a single figure corresponding to the area becomes immediately obvious as soon as sequential readings are studied.

\section{METHODS}

All individuals have been studied using a Bjerrum screen at 2.0 metres. A white test object $16 \mathrm{~mm}$ in diameter has been consistently used. This rather large object was chosen so that the patient should have no doubt about its presence when it came into sight. The patients were all instructed to respond as soon as they saw the rim of it and not to wait until the whole of the test object had become visible. The subject has worn spectacles when appropriate-that is, when the wearing of glasses made the responses more consistent.

Patient fatigue would make it a self-defeating exercise to attempt to plot the blind spots by means of so many points along their border that a continuous line could be obtained from them. Because of this, we have plotted no more than eight points which provide four measurements-vertical, horizontal, and two diagonals (Fig. 1a). The lengths are then divided by two to give us four dimensions-namely, $a, b, c, d($ Fig 1b). The following formula is then applied to obtain the area of the octagon:

$$
\frac{(a+b)(c+d)}{\sqrt{ } 2}
$$




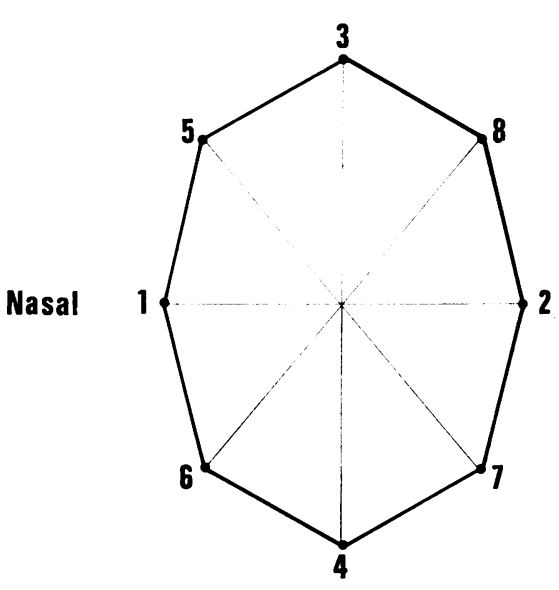

(a)

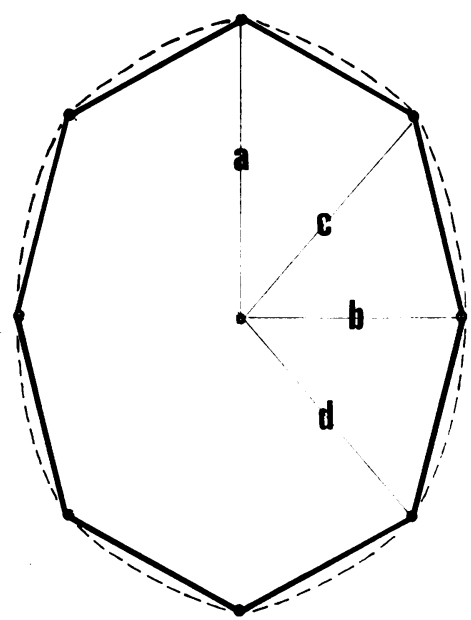

(b)
FIG. 1 (a) Order of plotting the eight points from which the blind spot area is calculated. (b) Half the horizontal, vertical, and diagonal dimensions are used in calculating the area of the blind spot.
We have found that this octagon provides an approximation to the true blind spot which has proved itself to be perfectly practicable. ${ }^{1}$

One of the essentials with a method employing serial measurements is to know that the findings are likely to be consistent. Accordingly, we made the simple experiment of studying the relationship between blind spot area and distance from the screen. If the technique is consistent the inverse square law should apply. Figure 2 shows that it does-within the limits of observer error. Because of the problems which could arise from slightly differing techniques, all the blind spots (with only four exceptions) have been charted by one individual (A.J.).

In order to obtain information about the 'normal' we studied a series of 30 individuals without complaints and with no specific ocular symptoms (this gave us a total of 53 normal eyes). The mean blind spot area was 67.5 sq. in. (1 sq. in. $=6.45 \mathrm{~cm}^{2}$ ). The range of blind spot area which we have accepted as normal was obtained by taking twice the standard deviation ( $S D=13.3$ sq. in.) on either side of the mean. Thus we concluded that areas between 41 and 94 sq. in. could be considered normal.

The patients included in this study have consisted of all those referred to one of us (A.J.) during the past 17 years in whom bilateral (and approximately symmetrical) papilloedema was observed in the

\footnotetext{
1 It may be that it would have been equally satisfactory to employ the formula $\pi \cdot r^{1} \cdot r^{2}$ for an elipse. We rejected this possibility because the blind spot may be irregular and it seemed to us pretentious to apply a formula which assumed the existence of more than the eight points which were actually measured. In fact, it seems to make very little practical difference which formula is applied to any one patient.
}

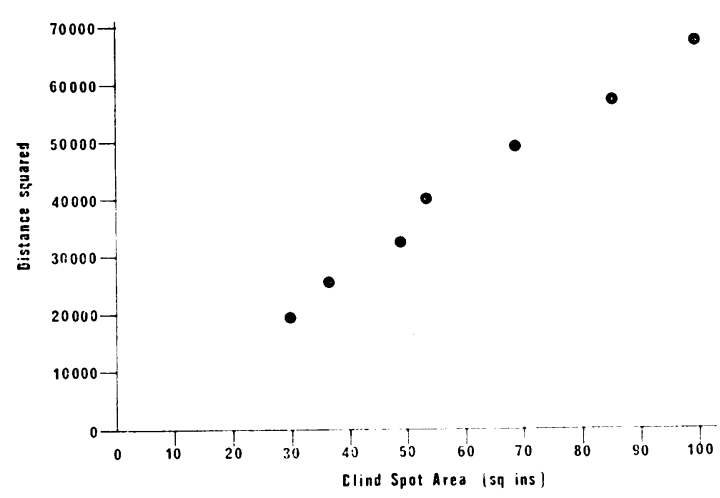

FIG. 2 Area of the blind spot which for one normal individual was recorded at seven different distances. Note: Ordinate $=$ square of distance $(\mathrm{cm}) ;$ abscissa $=$ blind spot area.

absence of any intracranial pathology. Under this heading we scrutinised the notes of 62 patients; 10 of these had to be excluded from further consideration merely because of insufficient data. We have classified and tabulated the remaining 52 patients (Table 1) so that the reader can see the background from which we have drawn the patients with whom this report is concerned. We have excluded children from this study, firstly, because attempts to measure their blind spots have not shown consistent results. Secondly, we suspect that papilloedema which accompanies children's febrile illnesses has a different pathogenesis, because it disappears very 
rapidly-for example, in two to four weeks-after resolution of the primary illnesses. These children often received no treatment other than antibiotics.

This paper is concerned with the group of 30 adult patients suffering from papilloedema without a detectable cause.

\section{TABLE 1}

ANALYSIS OF TOTAL CASE MATERIAL FROM WHICH 30 PATIENTS WITH BENIGN INTRACRANIAL HYPERTENSION WERE DRAWN

\begin{tabular}{|c|c|c|}
\hline & \multicolumn{2}{|c|}{ Number of patients } \\
\hline & Male & Female \\
\hline $\begin{array}{l}\text { Insufficient data } \\
\text { Children (under } 16 \text { years) }\end{array}$ & 2 & 8 \\
\hline $\begin{array}{l}\text { a. Classical otitic hydrocephalus } \\
\text { b. Upper respiratory or other infection } \\
\text { c. No obvious cause } \\
\text { d. Steroid administration }\end{array}$ & $\begin{array}{l}2 \\
3 \\
1 \\
1\end{array}$ & $\begin{array}{l}2 \\
2 \\
3 \\
0\end{array}$ \\
\hline $\begin{array}{l}\text { Adults } \\
\text { a. Classical otitic hydrocephalus } \\
\text { b. Mistaken diagnosis: } i . e ., \text { fundi } \\
\text { abnormal but not papilloedematous } \\
\text { c. Unclassified } \\
\text { d. Papilloedema of unknown origin } \\
\text { (benign intracranial hypertension) }\end{array}$ & $\begin{array}{l}1 \\
2 \\
1 \\
2\end{array}$ & $\begin{array}{l}0 \\
4 \\
0\end{array}$ \\
\hline
\end{tabular}

The age and sex distribution of these patients is set out in Fig 3. This shows that the patients were predominantly female and that although almost half of them were aged less than 26 years the older age groups were also involved.

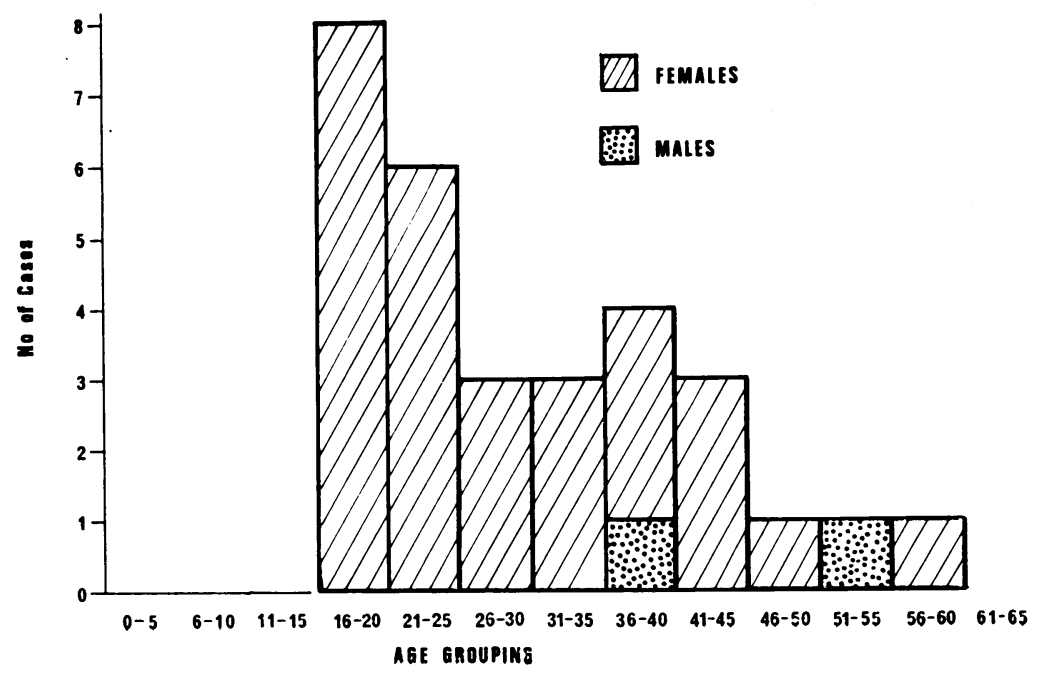

The diagnosis of non-tumour papilloedema was substantiated in some patients after special neuroradiological investigations-for example, angiography or pneumoencephalography-but in the remaining patients the diagnosis was made on clinical grounds aided by the echogram, the EMI scan, the gamma-scan, and by plain radiographs of the skull.

The patients with whom this report is chiefly concerned received treatment according to the methods listed in Table 2. The majority of the female patients were overweight (mean weight $=$ $78 \mathrm{~kg}$ ), and in such patients the therapy listed has been combined with a reducing diet.

TABLE 2

\section{ANALYSIS OF THERAPY USED IN MANAGEMENT OF} BENIGN INTRACRANIAL HYPERTENSION

\begin{tabular}{lr}
\hline Sole or chief therapy & Number of patients \\
\hline Subtemporal decompression & \\
(only for patients treated before 1958) & 5 \\
Dehydration therapy & 25 \\
Steroids & 0 \\
\hline
\end{tabular}

\section{RESULTS}

After the chosen therapy was established the patients were reviewed at intervals in the outpatient clinic until it became clear that the papilloedema had resolved completely. Two
FIG. 3 Age and sex distribution of 30 patients with papilloedema. 
females have suffered a second 'attack' of this disorder; two years and three years, respectively, separated the two episodes.

The preferred treatment has altered with the passage of time. Initially, before it was realised how effective dehydration therapy could be, we relied upon a subtemporal decompression to save the eyesight. This was a time-honoured and effective technique, but it has the disadvantage that it has now been shown to be unnecessary and the operated patients frequently continued with an unsightly (and sometimes tender) bulge in the decompression area for many years.

The dehydrating agent has been changed over the years and Table 3 lists the methods used. All patients treated by dehydration have been instructed to limit their daily fluid intake to $900 \mathrm{ml}$. Most of the patients treated with hydroflumethiazide and with chlorthalidone have been given potassium supplements.

\section{TABLE 3}

\section{ANALYSIS OF VARIOUS DEHYDRATING AGENTS USED}

\begin{tabular}{lc}
\hline \multicolumn{1}{c}{ Sole or chief dehydrating agent } & Number of episodes* \\
\hline Oral urea $(1.0 \mathrm{~g} / \mathrm{kg} / \mathrm{day})$ & 1 \\
Oral glycerol $(1.0-1.5 \mathrm{~g} / \mathrm{kg} /$ day $)$ & 7 \\
Hydroflumethiazide $(100 \mathrm{mg}$ alternate days) & 8 \\
Chlorthalidone $(200 \mathrm{mg}$ alternate days) & 11 \\
\hline
\end{tabular}

* There were 27 'episodes' among 25 patients not subjected to surgery.

The patients' blind spots were calculated according to the formula already described and the results of treatment have been plotted. Examples of the findings are shown in Figs. 4, $5,6,7,8$, and 11 . The shaded area bounded by horizontal dotted lines near the bottom of each graph indicates the range of the normal blind spot area. In this, and in all subsequent graphs, filled circles refer to the right eye, open circles to the left one. The rectangle marked 'pregnancy' terminated with delivery. The duration and nature of the therapy used is indicated by rectangles marked in the upper part of the charts. The patient's weight in $\mathrm{kg}$ is shown at intervals by the figures above the small black triangles.

Figure 4 shows the effectiveness of a subtemporal decompression. The vision of the left eye was never affected, but that of the right eye was $6 / 12$ initially and was restored to $6 / 6$ at the end of the therapy. However, this patient was left with a persistent, unsightly bulge in the subtemporal region which was still present when she was seen for another reason 14 years later.

Figure 5 shows that by using oral urea an effect similar to that which results from surgical decompression is eventually achieved. In this patient it will be noticed that a relapse followed the cessation of treatment and an additional course of urea was necessary.

Figure 6 shows the most dramatic response which we observed after the use of oral glycerol. This patient was admitted to hospital with a history of visual failure which had developed during the preceding eight weeks. For three weeks a left external rectus weakness had been present. The graph shows that the papilloedema had virtually disappeared at the end of two months. Her visual acuities at the end of treatment were $6 / 9$ and Jaeger 1 bilaterally. The resolution of papilloedema when using glycerol was not always so prompt or so complete.

Figure $6 \mathrm{~b}$ illustrates an example where very little progress was achieved by glycerol but eventual resolution accompanied treatment with hydroflumethiazide.

Figure 7a shows the best response seen when using hydroflumethiazide, while Fig. $7 \mathrm{~b}$ shows a less impressive result, but one which is of interest because a relapse followed when the treatment was stopped before the papilloedema had fully resolved.

Figure 8 shows a striking resolution of the enlarged blind spots in a young pregnant girl who presented with visual failure. At the start of treatment this girl's visual acuities were 6/9 right and 6/24 left. After only four days treatment her acuities had already improved to $6 / 6$ right and 6/18 left. Six days later-that is, only 10 days after the dehydration regimen had been instituted-the visual acuities were $6 / 6$ bilaterally and N,5 right, N,8 left. The weight gain $(79.0 \mathrm{~kg}-80.6 \mathrm{~kg})$ in the Figure is presumably related to pregnancy because this patient lost $2 \mathrm{~kg}$ in her first week in hospital which suggested that she had an excess of body water of which she rid herself promptly in response to treatment. Figure $8 \mathrm{~b}$ shows her right optic disc four days after therapy began, and Fig. 8C shows the appearance six weeks later. 


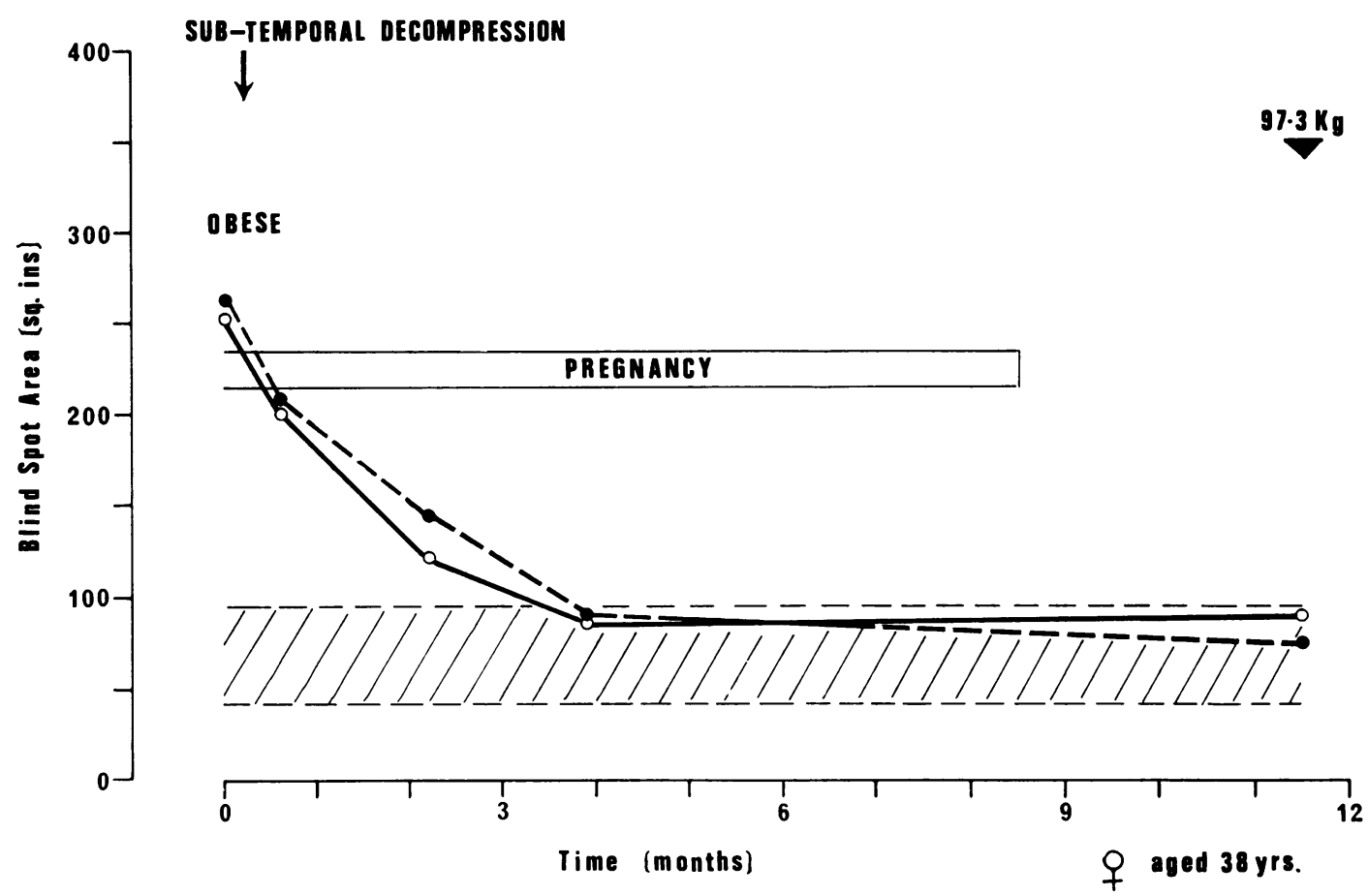

FIG. 4 Blind spot areas of a patient whose papilloedema was treated by subtemporal decompression. Note that there was still a considerable enlargement of the blind spot two months after the operation.

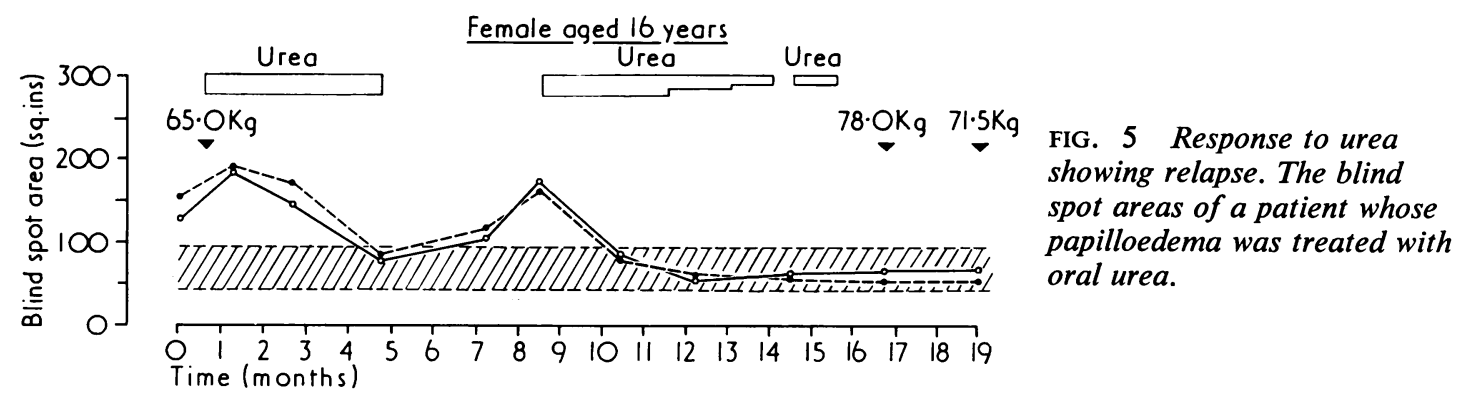

It can be seen that the papilloedema had resolved completely by the end of six weeks, but optic atrophy had developed. In spite of the optic atrophy, the visual acuity was fully preserved at $6 / 5$.

Through the courtesy of Mr Ian Strachan, fluorescein angiograms have been performed on a number of our patients. These were carried out to help record the progress of the disease. Figure 9 shows the appearances, early and late, after the intravenous injection of $2.5 \mathrm{ml} 25 \%$ fluorescein. The figure also includes our measurements of the blind spot area. The particular pairs of photographs would seem to indicate some correlation between the severity of the abnormalities and the size of the blind spot; however, we have insufficient data to say more than this.

Figure 10 shows how clearly fluorescein angiography demonstrates the resolution of the papilloedema at the conclusion of treatment. 


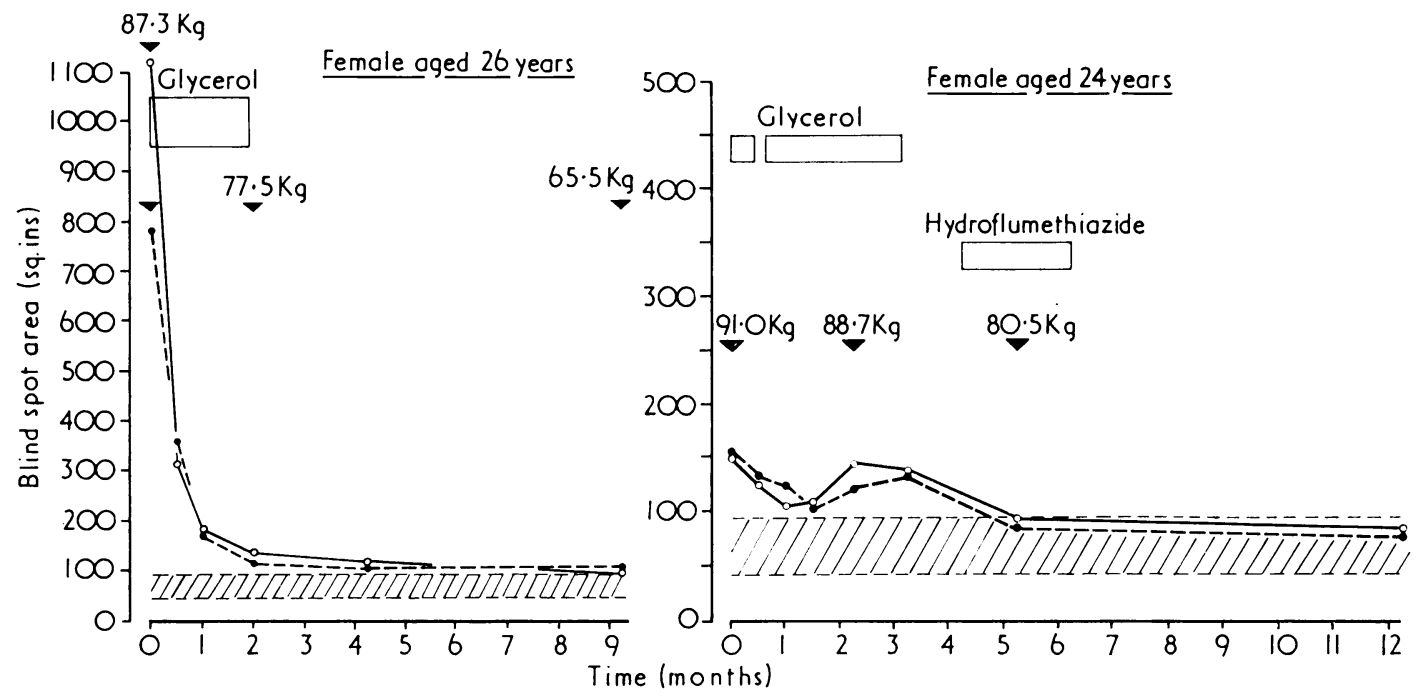

FIG 6 (a) Response to glycerol. The blind spot areas of a patient whose papilloedema was treated with oral glycerol $(1.5 \mathrm{~g} / \mathrm{kg} /$ day $)$. Note that in this patient the blind spots never receded to within the normal range. (b) Response to glycerol showing relapse. Blind spot areas of a patient who did not respond satisfactorily to oral glycerol and who was eventually. cured by hydroflumethiazide.

FIG. 7 The blind spot areas of patients whose papilloedema was treated with hydroflumethiazide $100 \mathrm{mg}$ every second day (see text): (a) response, (b) response with relapse. Note in (a) how at the end of treatment the blind spot areas remain at the upper limit of normal. Incidentally, the consistency of the measurement once the papilloedema had resolved confirms the validity of the method. 


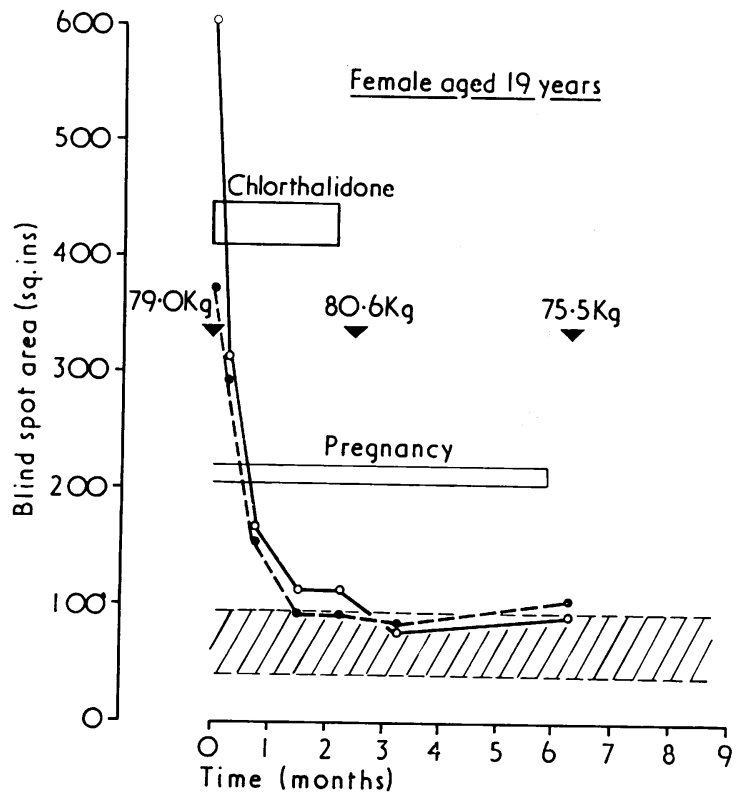

FIG. 8 (a) Response to chlorthalidone. The blind spot areas of a young pregnant girl with visual impairment arising from papilloedema. Note that the blind spots are almost normal after only six weeks.

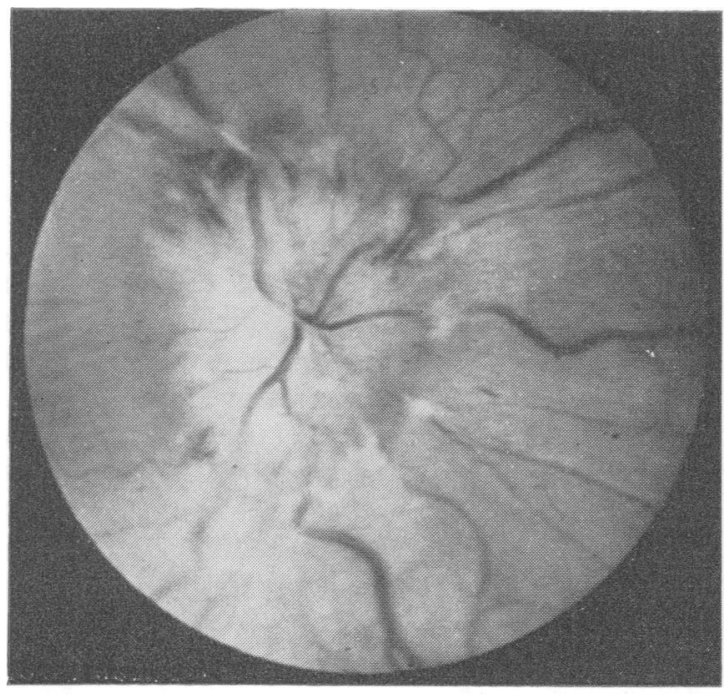

(b)

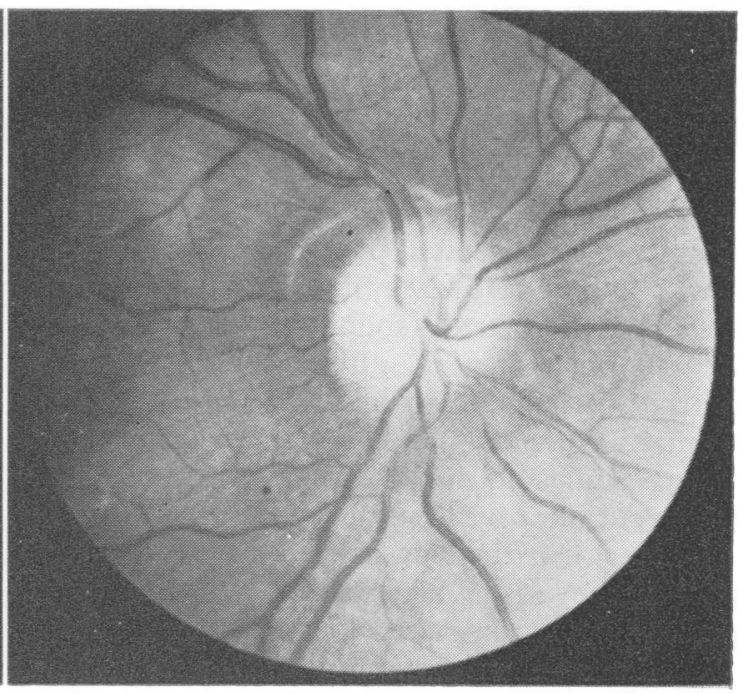

(c)

FIG. 8 (b) Same patient's right optic disc after four days' therapy: the blind spot at this stage measured 333 sq in. (c) The same, six weeks later. The blind spot was now 93 sq. in.

We have attempted to relate the area of pathology outlined by the late fluorescence of the disc to the size of the blind spot. However, we have to report that in the few cases where this was possible there was no consistent relationship between these areas.

It was mentioned above that vision may frequently become seriously impaired in this disorder. Indeed, in a series of nine untreated patients whose notes have been traced in the 

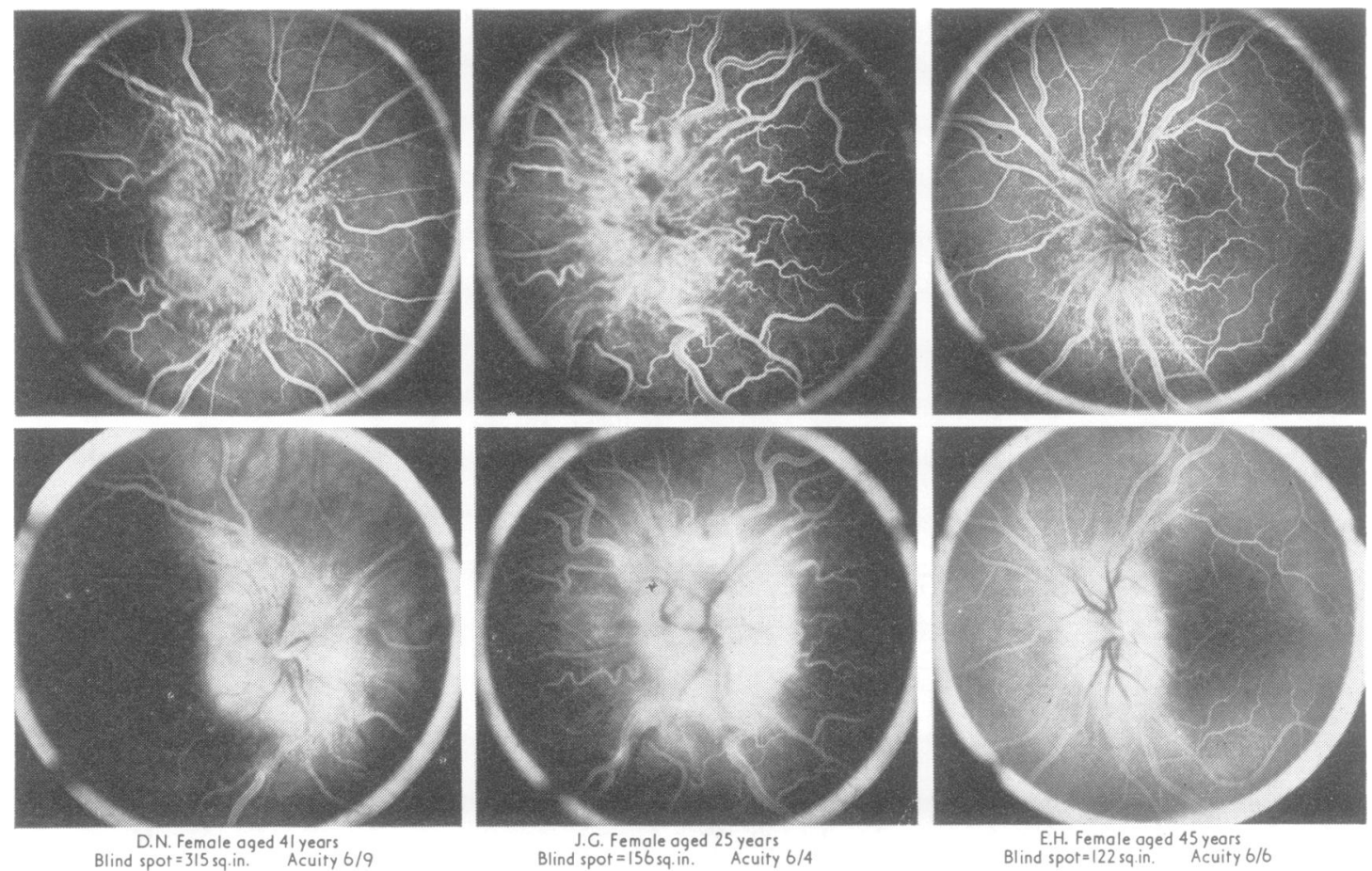

FIG. 9 Fluorescein angiograms of three patients; arterial phase above; venous phase below. See text.

records of this hospital, two (22\%) progressed to near blindness. However, it appears that prompt therapy is consistently followed by improvement in the visual acuities, although the eyes may remain permanently slightly damaged by the disease. For example, one patient had initial visual acuities of 6/12,6/36 and her acuities after treatment improved only to $6 / 9,6 / 12$.

Table 4 lists the data concerning the pre- and post-therapy visual acuities in all those patients presenting with impaired vision. (The remaining 17 patients-those presenting with visual acuity $6 / 6$ or better-continued in the same category, although two of them showed a post-treatment acuity of $6 / 6$ as compared with a pre-treatment $6 / 5$ ). There were only two patients whose vision did not recover to at least $6 / 9$; one of them had had a diagnosis of 'lazy eye' established many years earlier. The fact that this type of papilloedema may be associated with some residual tissue damage would explain our observation that, at the end of treatment, when the papilloedema had fully resolved, not all of our patients had blind spot areas within the 'normal' range. This observation may well be related to the appearance of optic atrophy which is sometimes noticeable at the conclusion of treatment even though visual acuities are preserved ( $c f$. Fig. 8c). Our data are not sufficiently large to let us draw absolutely firm conclusions, but it appears that the patients whose blind spots are initially much enlarged-for example, over 300 sq. in.-are those most likely to show some permanent increase in blind spot size.

It is sometimes difficult satisfactorily to assess the duration of treatment required to obtain complete resolution of the papilloedema-that is 'time-to-cure'. This difficulty has arisen because the patients at this stage are being seen at three to four week intervals as outpatients and no information is obtained between visits. Sometimes also, in the earlier part of this study, a patient would have the treatment changed because the first agent used had been seen to be ineffective; in such a situation one obviously could not speak of a 'time-to-cure'. We have 

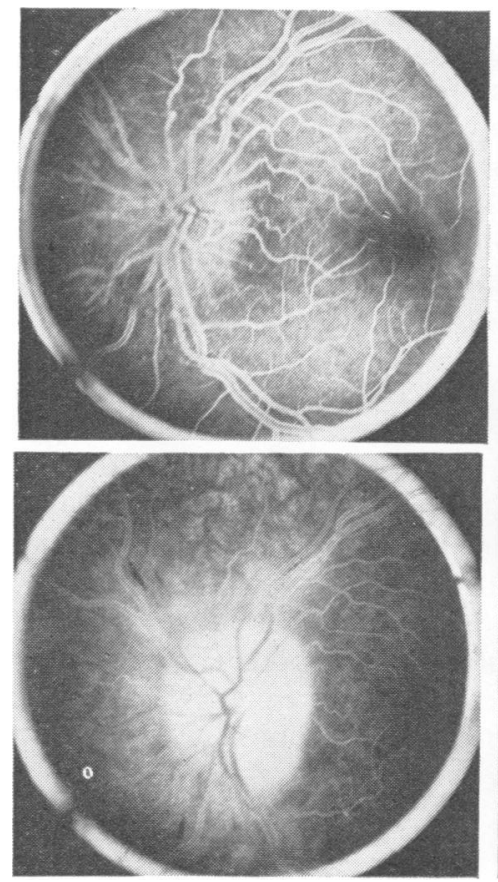

Before treatment

Blind spot $=181$ sq. in. Acuity $6 / 4$
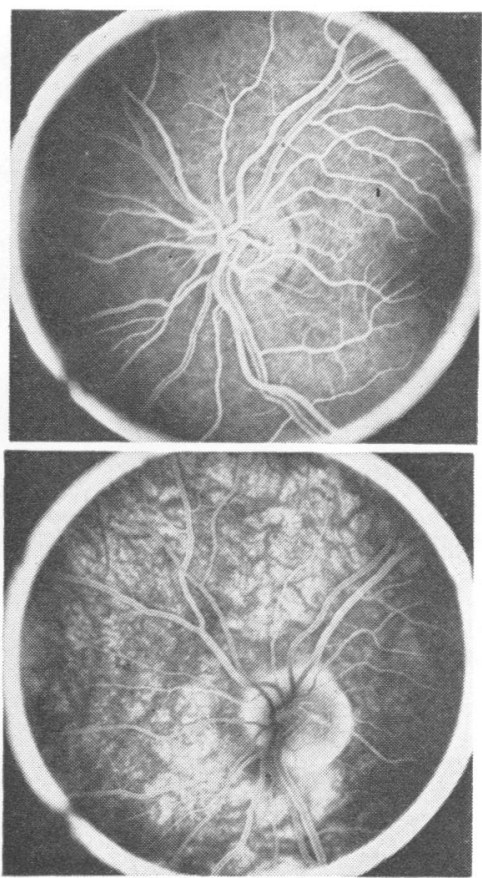

After 6 wk treatment with chlorthalidone Blind spot $=71$ sq. in.
FIG. 10 Fluorescein angiograms of female, aged 16 years. Arterial phase above; venous phase below. Left, before and, right, after treatment.

TABLE 4

TABULATION OF VISUAL ACUITIES BEFORE AND AFTER TREATMENT OF ALL THOSE PATIENTS WHO PRESENTED WITH SOME VISUAL IMPAIRMENT

\begin{tabular}{|c|c|c|c|c|c|c|}
\hline \multirow[t]{2}{*}{ Patient } & \multicolumn{3}{|c|}{ Initial acuities } & \multicolumn{3}{|c|}{ Final acuities } \\
\hline & $R$ eye & Leye & $\begin{array}{c}\text { Wearing } \\
\text { correcting } \\
\text { lenses }\end{array}$ & $R$ eye & Leye & $\begin{array}{c}\text { Wearing } \\
\text { correcting } \\
\text { lenses }\end{array}$ \\
\hline $\begin{array}{l}\text { S.C. } \\
\text { E.G. } \\
\text { K.L. } \\
\text { P.G. } \\
\text { N.G. } \\
\text { I.H. } \\
\text { P.M. } \\
\text { M.D. } \\
\text { D.N. (1) } \\
\text { D.B. } \\
\text { J.F. } \\
\text { D.N. (2) } \\
\text { P.N. } \\
\text { P.P. }\end{array}$ & $\begin{array}{l}6 / 9 \\
6 / 9 \\
6 / 36^{*} \\
6 / 12 \\
6 / 9 \\
6 / 6 \\
6 / 9^{*} \\
6 / 6 \\
6 / 9 \\
6 / 9 \\
6 / 12 \\
6 / 6 \\
6 / 12 \\
6 / 6\end{array}$ & $\begin{array}{l}6 / 7.5 \\
6 / 6 \\
6 / 36 \\
6 / 36 \\
6 / 12 \\
6 / 24^{*} \\
6 / 4 \\
6 / 9 \\
6 / 9 \\
6 / 24 \\
6 / 7.5 \\
6 / 9 \\
6 / 4 \\
6 / 9\end{array}$ & $\begin{array}{l}+ \\
+ \\
+\end{array}$ & $\begin{array}{l}6 / 6 \\
6 / 4 \\
6 / 36^{*} \\
6 / 9 \\
6 / 6 \\
6 / 5 \\
6 / 9^{*} \\
6 / 5 \\
6 / 6 \\
6 / 4 \\
6 / 5 \\
6 / 5 \\
6 / 6 \\
6 / 4\end{array}$ & $\begin{array}{l}6 / 6 \\
6 / 4 \\
6 / 6 \\
6 / 12 \\
6 / 9 \\
6 / 99^{*} \\
6 / 5 \\
6 / 6 \\
6 / 6 \\
6 / 5 \\
6 / 6 \\
6 / 6 \\
6 / 5 \\
6 / 4\end{array}$ & $\begin{array}{l}+ \\
+\end{array}$ \\
\hline
\end{tabular}

* Known lazy eyes.

(1) First occasion. (2) Second occasion. 
come to believe that chlorthalidone is superior to hydroflumethiazide because the mean 'timeto-cure' with chlorthalidone has worked out in 10 patients at 12 weeks. The rapidity and consistency with which the papilloedema has resolved in the group treated with chlorthalidone is shown in Fig. 11 in which the blind spot areas of 11 patients are superimposed on one chart.

\section{DISCUSSION}

Papilloedema occurring without tumour has been widely accepted as capable of causing serious visual impairment. For example, Foley (1955) reported permanent visual impairment in $23 \%$ of his cases with a similar type of papilloedema. Some untreated cases which we have culled from the records of this hospital, although few in number, provide a comparable figure

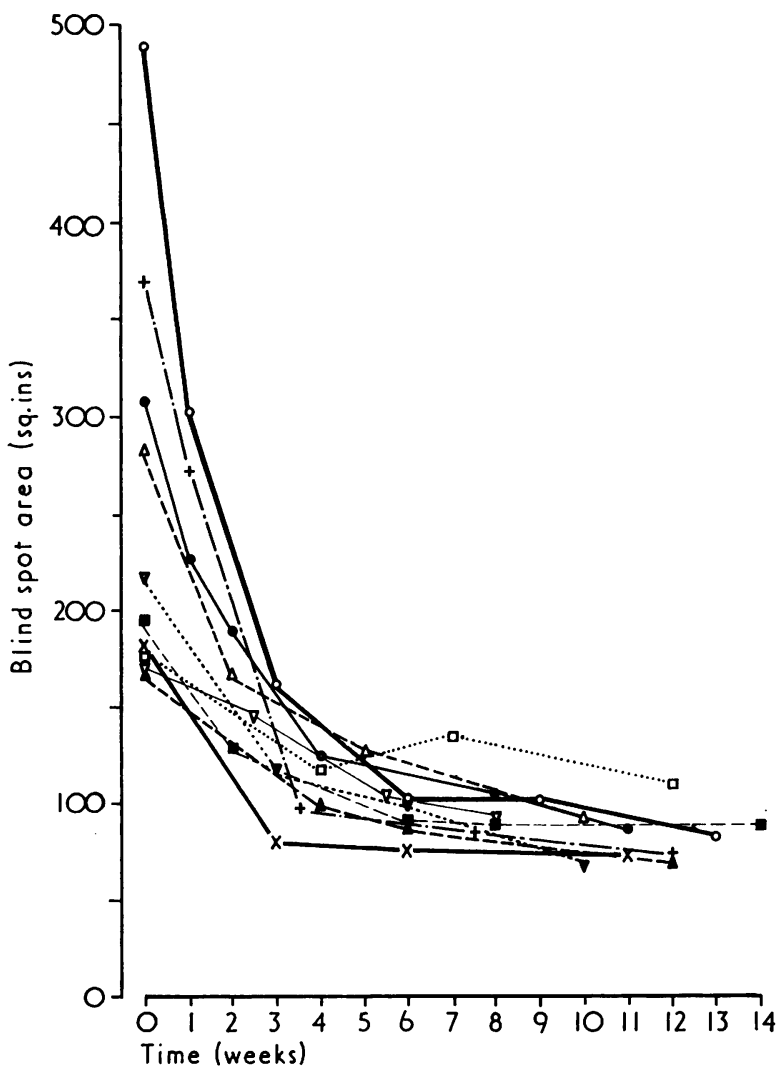

$(22 \%)$. If one accepts that there is every reason to take this condition (which is 'benign' as to life) very seriously then one should concentrate on devising the best method of treatment.

Over the years surgical methods have proved themselves to be effective. The neurosurgical techniques adopted have been unilateral or even bilateral subtemporal decompressions, posterior fossa decompression, or ventriculoatrial shunting.

All these methods are reported to be effective, although many authors from Dandy (1937) onwards have noted that with these patients the area of the subtemporal decompression sometimes remains bulging in an unsightly manner for many months or even years. More recently, following the experimental work of Hayreh (1964), there have been reports from ophthalmological surgeons who have effectively relieved the papilloedema by incising the optic nerve
FIG. 11 The blind spot areas of 10 patients treated with chlorthalidone. Note that the time scale differs from that used in earlier figures. In this figure the plot for each patient is compiled from the mean of the blind spot areas of the two eyes. 
sheath (see, for example, Davidson, 1969, or Galbraith and Sullivan, 1973).

In the literature (Paterson et al., 1961) the idea has developed that high doses of steroids are valuable in the management of this type of papilloedema. They have deliberately been avoided in our patients because it was quickly realised that dehydration was effective and dehydrating agents seemed less potentially dangerous than steroids. We have received personal communications (Winn, 1974; Hulme, 1975) in the form of tracings obtained during the continuous monitoring of intracranial pressure which show that in two young female patients suffering from this syndrome high doses of steroids did not lower the intracranial pressure, whereas dehydrating agents did so.

With regard to the treatment, it may be that satisfactory loss of weight by the obese female is an important factor associated with the resolution of the papilloedema. Certainly the factor of obesity when combined with being female has been a consistent presenting feature among an otherwise assorted group. Various authors (e.g. Greer, 1965) have drawn attention to this previously. Perhaps obesity has also been a factor, if not 'the' factor, underlying the rare familial occurrence of this disorder (Howe et al., 1973).

We started with the notion that this syndrome was seen chiefly in the overweight young female with a recent pregnancy or with some menstrual disorder. Our own material certainly shows that the young female is more frequently affected than is the older woman ( $c f$ Fig. 3). Perhaps the belief that recent pregnancy or a menstrual disorder is an important factor arises from nothing more than the fact that both these events are fairly common in the younger female (see also Johnston and Paterson, 1974). Certainly, we no longer regard these features as a relevant part of the syndrome. However, we do consider that there is a metabolic element which underlies this condition and in this respect our theorising follows along one or more of the trails embarked upon by Thomas (1933) and by Oldstone (1966).

Although this syndrome predominantly affects the female, a small proportion of male patients also suffer from papilloedema without an intracranial mass ( $c f$. Dandy, 1937; also Boddie et al., 1974). With only two male patients we can have no views as to whether the syndrome in the male differs in any important aetiological factors from the one seen in the female. Furthermore, even in the female, we have no certain knowledge that this syndrome encompasses an homogeneous entity.

We must emphasize that we have rejected from consideration all patients with an infective element in their presentation-that is, all classical and not-so-classical examples of 'otitic hydrocephalus'-also all patients with monocular or widely asymmetrical papilloedema. It will be recalled that Table 1 demonstrated this in detail. With regard to the entry 'Mistaken diagnosis' in that table, we have found the advent of fluorescein angiography (e.g. Miller et al., 1965, also Rosen, 1969) of very great value. Occasions on which we would in the past have prescribed treatment and followed up patients for many months can be eliminated by the judicious use of fluorescein coupled when necessary with stereoscopic colour photographs of the disc. Because fluorescein angiography distresses some patients, we would hold back from recommending it routinely. However, if the clinical presentation is unusual, or the appearance of the fundi not really typical, or the blind spots are not initially greatly enlarged-for example, no more than about 120 sq. in.-these patients should be referred for fluorescein studies. When used, fluorescein should be employed early because, in our experience, resolving papilloedema may very easily be mistaken for drusen of the disc. Thus, fluorescein used late may serve to confuse and not to clarify the issue.

With regard to the measurement of the blind spots, a study of the literature suggests that there has been very little systematic usage of blind spot size as a means of recording the severity of papilloedema since Loyal Davis (1929) reported his findings. He was, of course, recording the resolution of papilloedema after the excision of intracranial masses. So far as we are aware, there are no published reports of blind spot size being used as a means of monitoring the resolution of non-tumour papilloedema. Indeed, among the very few other clinicians interested in recording blind spot size as a matter of routine have been Benshtein (1961) and Ustinova (1962); while Shumilova (1962) observed a diminution 
in the size of the blind spot after dehydration of the hypertensive patient. Because of the apparent lack of general interest in blind spot measurement, we have concluded that it is not generally realized how easily these measurements may be obtained in any cooperative subject, nor is it appreciated how consistent they are. The graphs reproduced in the present paper speak for themselves as regards consistency. (It must be recalled that all the plots were obtained by one observer, but two or more equally experienced observers might well find that they obtained closely comparable if not identical results). We heartily commend the use of serial measurements of the blind spot to all those who have the care of this group of patients, and we are sure that such measurements are essential in the effective management of non-tumour papilloedema.

In recommending the measurement of the blind spots as a means of following the resolution of papilloedema, we must point out that there is not a $1: 1$ correlation at the beginning and at the end of treatment between the area of the blind spot and the area containing visible abnormalities on the fluorescein angiogram. It may very well be that the pathological features which enlarge the blind spot include damage to the sensory layer of the retina which is not necessarily visible to the ophthalmoscope. The fact that the blind spots have not always returned to normal in our treated patients could be the result of permanent peri-papillary damage, or alternatively could be a manifestation of the optic atrophy which we have sometimes noted-for example, Fig. 8c.

We have already referred to difficulties in diagnosis with specific regard to the optic fundi, but it is necessary to allude briefly to the clinical diagnosis. The criteria we have followed have been as follows. The clinical picture must be one of raised intracranial pressure only-that is, with the exception of a mildly unsteady gait, there must be no abnormal neurological signs except either VIth nerve palsy (unilateral or bilateral) or those relating to the papilloedema itself. The radiographs of the skull in children may show suture separation, but in adults we would advise against accepting the diagnosis of non-tumour papilloedema if there are any indications of chronic raised intracranial pres- sure. For example, erosion of the pituitary fossa is a feature of long-standing raised intracranial pressure and simply will not be found in conjunction with a relatively short history (in this series its mean duration was 14 weeks), which suggests that this type of papilloedema develops over a short period of time. In a few patients (for example, Fig. 8 above) the history was as short as three weeks and we believe that this very short history has a diagnostic value when one is confronted by a patient with flagrant papilloedema and no other abnormal neurological signs except for those which could result from raised intracranial pressure. Apart from plain radiographs (and always provided that the above-mentioned clinical criteria are strictly followed) the diagnostic investigations which will be necessary from now on are as follows. In a department with an ultrasonic A-scan which is deployed with reliable accuracy this investigation alone is of great value. When a patient with florid papilloedema can be shown by the A-scan to have a central undilated 3 rd ventricle $\stackrel{\circ}{\circ}$ one knows that if the result is correct then an intracranial mass has virtually been excluded. However, since so much is at stake for the patient, a gamma-scan has often also beenco performed. In the future a CAT scan (EMI scan) will probably become the final arbiter in the diagnosis of these patients and its use should obviate the need for both angiography and for delineation of the ventricles.

In the literature, a restricted fluid intake, a salt free diet, diuretics, and acetazolamide have been shown to have a place in the treatment of benign intracranial hypertension. The contribution of the present writers is to have stated categorically that surgery is not required in the treatment of this condition and to stress that this holds true even when the vision has begun to fail. Although our results show that a certain amount of optic atrophy frequently follows an advanced degree of papilloedema in this condition, there appears to be so much 'spare capacity' among the optic nerve fibres that in practice and with very few exceptions the visual acuities at the end of therapy have either been maintained intact, or have substantially recovered.

The message of our work is to stress that dehydration therapy alone is sufficient treatment for this condition. Chlorthalidone seems to 
be the best agent that we have employed to date, but we hold no special brief for it as a permanent or inevitable first choice. One of the difficulties in finding the most effective therapy is that these patients are generally so fit and well that we have often seen no need to admit them to hospital. Even when they have been inpatients the propriety of inserting an intracranial pressure measuring device in order to study the comparative efficacy of various dehydrating agents must be in doubt, so that, with a relatively rare condition (only 30 patients in 18 years), also a condition in which 'invasive' measuring techniques may be unacceptable on ethical grounds, progress towards the selection of the perfect dehydrating agent may have to be slow. However, we would suggest that slow progress in this respect is not vitally important because all the forms of therapy used in this series have been effective in saving vision. Once reasonably efficient dehydration has been set in motion, it appears that vision will be preserved provided that treatment is not stopped before the condition has resolved. This correlates with the fact, well known to surgeons who practised the method, that, although subtemporal decompression was effective in preserving vision, it did not always resolve the papilloedema rapidly.

We would like to record our thanks to the Ryder Briggs Memorial Foundation for financial assistance to one of us (J. C.) during the preparation of this paper. We are also grateful to $\mathrm{Mr} \mathbf{A}$. L. Foster for his skill and care in preparing the graphs, and to Mrs M. Saynor, who took the fundus photographs.

\section{REFERENCES}

Benshtein, I. Yu. (1961). On the method of measuring the blind spot area. (Russian). Vestnik Oftalmoloqii, 74, 45-46.

Boddie, H. G., Banna, M. M., and Bradley, W. G. (1974). 'Benign' intracranial hypertension. A survey of the clinical and radiological features, and long term prognosis. Brain, 97, 313-326.

Dandy, W. E. (1937). Intracranial pressure without brain tumour. Annals of Surgery, 106, 492-513.

Davidson, S. I. (1969). A surgical approach to plerocephalic disc oedema. Transactions of the Opthalmological Society of the U.K., 89, 669-690.

Davis, L. (1929). The blind spots in patients with intracranial tumours. Journal of the American Medical Association, 92, 794-798.

Foley, J. (1955). Benign forms of intracranial hypertension: 'toxic' and 'otitic' hydrocephalus. Brain, 78, 1-41.

Galbraith, J. E. K., and Sullivan, J. H. (1973). Decompression of the perioptic meninges for relief of papilledema. American Journal of Opthalmology, 76, 687-692.

Greer, M. (1965). Benign intracranial hypertension. 6. Obesity. Neurology (Minneap.), 15, 382-388.

Hayreh, S. S. (1964). Pathogenesis of oedema of the optic disc. British Journal of Opthalmology, 48, 522-543.

Howe, J. G., Saunders, M., and Clarke, P. R. R. (1973). Familial benign intracranial hypertension. Acta Neurochirurgica (Wien), 29, 173-175.

Johnston, I., and Paterson, A. (1974). Benign intracranial hypertension. I. Diagnosis and prognosis. Brain, 97, 289-300.

Miller, S. J. H., Sanders, M. D., and Ffytche, T. J. (1965). Fluorescein fundus photography in the detection of early papilloedema and its differentiation from pseudo-papilloedema. Lancet, 2, 651-654.

Oldstone, M. B. (1966). The endocrinological aspects of benign intracranial hypertension Archives of Neurology (Chic.), 15, 362-366.

Paterson, R., de Pasquale, N., and Mann, S. (1961). Pseudotumor cerebri. Medicine (Balt.), 40, 85-99.

Rosen, E. S. (1969). Fluorescence Photography of the Eye, pp. 345. Butterworths: London.

Shumilova, V. G. (1962). Changes in the blind spot and ocular fundus in arterial hypertension. (Russian). Soviet Medicine, 25, 147-150.

Thomas, W. A. (1933). Generalized edema occurring only at the menstrual period. Journal of the American Medical Association, 101, 1126-1127.

Ustinova, E. I. (1962). On the method of registration and measurement of the blind spot. (Russian). Vestnik Oftalmoloqii, 75, 49-51. 